\title{
Plasma circulating tumor DNA assessment reveals $K M T 2 D$ as a potential poor prognostic factor in extranodal NK/T-cell lymphoma
}

Qiong $\mathrm{Li}^{1,2+}$, Wei Zhang ${ }^{1,2 \dagger}$, Jiali $\mathrm{Li}^{1,2}$, Jingkang Xiong ${ }^{1,2}$, Jia Liu ${ }^{1,2}$, Ting Chen ${ }^{1,2}$, Qin Wen ${ }^{1,2}$, Yunjing Zeng ${ }^{1,2}$, $\mathrm{Li} \mathrm{GaO}^{1,2}$, Lei Gao ${ }^{1,2}$, Cheng Zhang ${ }^{1,2}$, Peiyan Kong ${ }^{1,2}$, Xiangui Peng ${ }^{1,2}$, Yao Liu ${ }^{1,2^{*}}$, Xi Zhang ${ }^{1,2^{*}}$ and Jun Rao ${ }^{1,2^{*}}$ (D)

\begin{abstract}
Background: The early detection of tumors upon initial diagnosis or during routine surveillance is important for improving survival outcomes. Here, we investigated the feasibility and clinical significance of circulating tumor DNA (ctDNA) detection for Extranodal NKTT-cell lymphoma, nasal type (ENTKL).

Methods: The plasma ctDNA assessment was based on blood specimens collected from 65 newly diagnosed patients with ENKTL in the hematology medical center of Xinqiao Hospital. Longitudinal samples collected under chemotherapy were also included. The gene mutation spectrum of ENKTL was analyzed via next generation sequencing.
\end{abstract}

Results: We found that the most frequently mutated genes were KMT2D (23.1\%), APC (12.3\%), ATM (10.8\%), ASXL3 (9.2\%), JAK3 (9.2\%), SETD2 (9.2\%), TP53 (9.2\%) and NOTCH1 (7.7\%). The mutation allele frequencies of ATM and JAK3 were significantly correlated with the disease stage, and mutated KMT2D, ASXL3 and JAK3 were positively correlated with the metabolic tumor burden of the patients. Compared with the tumor tissue, ctDNA profiling showed good concordance (93.75\%). Serial ctDNA analysis showed that treatment with chemotherapy could decrease the number and mutation allele frequencies of the genes. Compared with PET/CT, ctDNA has more advantages in tracking residual disease in patients. In addition, patients with mutated KMT2D had higher expression compared with those with wild type, and mutated KMT2D predicted poor prognosis.

Conclusion: Our results unveil the mutation spectrum of ENKTL patients' plasma, which can be used to monitor the disease status of the patients exactly, and KMT2D is the most frequently mutated gene with prognosis prediction value. The application of ctDNA sequencing can provide precision treatment strategies for patients.

Trial registration: This study is registered with chictr.org (ChiCTR1800014813, registered 7 February, 2018Retrospectively registered).

Keywords: ENKTL, Circulating tumor DNA, Mutation allele frequency, Minimal residual disease, Prognosis

\footnotetext{
*Correspondence: 648283926@qq.com; zhangxxi@sina.com; raojun1129@126.com

${ }^{\dagger}$ Qiong Li and Wei Zhang contributed equally to this work.

${ }^{1}$ Medical Center of Hematology, Xinqiao Hospital, Army Medical University, Chongqing, China

Full list of author information is available at the end of the article
}

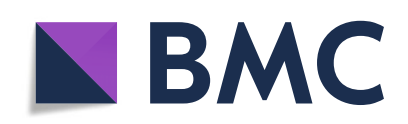

(- The Author(s). 2020 Open Access This article is licensed under a Creative Commons Attribution 4.0 International License, which permits use, sharing, adaptation, distribution and reproduction in any medium or format, as long as you give appropriate credit to the original author(s) and the source, provide a link to the Creative Commons licence, and indicate if changes were made. The images or other third party material in this article are included in the article's Creative Commons licence, unless indicated otherwise in a credit line to the material. If material is not included in the article's Creative Commons licence and your intended use is not permitted by statutory regulation or exceeds the permitted use, you will need to obtain permission directly from the copyright holder. To view a copy of this licence, visit http://creativecommons.org/licenses/by/4.0/. The Creative Commons Public Domain Dedication waiver (http://creativecommons.org/publicdomain/zero/1.0/) applies to the data made available in this article, unless otherwise stated in a credit line to the data. 


\section{Introduction}

Extranodal NK/T-cell lymphoma, nasal type (ENTKL), is an aggressive extranodal lymphoma of NK-cell or T-cell lineage, which is highly aggressive and heterogeneous disease, with predominance in males [1-4]. Current treatment strategies (such as the combination of chemotherapy, radiotherapy, targeted therapy) can improve the complete remission of patients, but most will ultimately relapse and progress [5-7]. For these patients, standard monitoring methods, including computed tomography (CT) and serum protein markers analysis upon initial diagnosis and during routine surveillance, are pivotal for therapeutic response evaluation; however, these tests have limitations of low sensitivity and specificity or false positivity. Therefore, there is an urgent need for a highly sensitive, standardized and noninvasive assay that can be used for detecting early relapse and/or progression of disease.

Current treatment response criteria for nonHodgkin lymphoma (non-HL) rely on CT scans or positron emission tomography (PET) scans. Imaging scans upon initial diagnosis or recurrence can provide macro overviews of the tumor volume and location, but $\mathrm{CT}$ scans have some limitations due to cost and radiation exposure, and PET lacks specificity [8-10]. Moreover, imaging scans cannot monitor dynamic tumor response and the clonal evolution in time. Therefore, clinically validated technology is needed to overcome the current monitoring treatment response limitations. One potential biomarker for detection is circulating cell-free DNA (cfDNA), which comes from dying cells that release DNA fragments into circulation, and in tumor patients, some of the cfDNA primarily originates from apoptosis and necrotic cancer cells, which carry tumor-specific alterations (termed circulating tumor DNA, ctDNA) [11-14]. ctDNA sequencing is a promising tool for the real-time monitoring of tumor progression, and its application has been explored in multiple solid tumors [15]. ctDNA can reflect the temporal evolution of tumors. Current studies have shown that ctDNA detection can be used to monitor minimal residual disease and track recurrence after treatment $[16,17]$. In addition, ctDNA sequencing is a noninvasive and tumor-specific assay, and ctDNA in circulation has a half-life of less than $2 \mathrm{~h}$, which is more sensitive than protein biomarkers and more specific than routine surveillance imaging through CT scans or PET scans [18]. Thus, dynamic tracking of ctDNA would be of higher value in guiding personalized management strategy.

The clinical application of ctDNA has been performed for multiple types of lymphoma, such as diffuse large Bcell lymphoma (DLBCL), classical HL, follicular lymphoma, multiple myeloma and peripheral T-cell lymphoma
[19-25]. ctDNA assessment acts as a dynamic marker of burden during treatment based on immunoglobulin next-generation sequencing (Ig-NGS), T-cell receptor gene next-generation sequencing (TCR-NGS) and cancer personalized profiling sequencing (CAPP-Seq). Recent evidence concerning ctDNA assessment in peripheral T-cell lymphoma by TCR-NGS suggested that ctDNA detection in T-lineage lymphoma is feasible [26]. In the present study, we thoroughly determined the molecular spectrum of ctDNA mutations in ENTKL and characterized the correlation between ctDNA mutations and clinical factors. Paired tumor tissues and plasma samples were collected to compare the concordance between tumor DNA and ctDNA. Moreover, dynamic ctDNA mutation alterations during treatment were also observed.

\section{Materials and methods}

\section{Study design and patient selection}

In this prospective cohort study, 65 patients newly diagnosed with ENKTL in the hematology medical center of Xinqiao Hospital from February 2017 to December 2019 were enrolled (ClinicalTrials identifier: ChiCTR1800014813). All consecutive patients who were deemed appropriate for this study during the study period were included without selection. Histological diagnoses were established independently by at least two experienced senior pathologists according to the WHO classification of Tumors of Hematopoietic and Lymphoid tissue criteria. All patients underwent baseline staging using laboratory, radiographic, and bone marrow examinations upon diagnosis. The Eastern Cooperative Oncology Group (ECOG) performance status was also assessed. The stage was evaluated in accordance with the Ann Arbor staging system. The International Prognostic Index (IPI) was calculated based on the serum lactate dehydrogenase, stage, extranodal status and performance status. Patient characteristics and treatment regimens of each therapy cycle were collected from each patient. Three healthy individuals were also recruited as control participants to test the accuracy of our sequencing platform for ctDNA profiling. All participants provided informed written consent before undergoing any study-related procedures in accordance with the Declaration of Helsinki. This study was approved by the China Ethics Committee of Registering Clinical Trials (ChiECRCT-20,180,005).

After the initial stage assessment, all patients were given 6 to 8 cycles of pegaspargase-based CHOP like regimens. Patients were reviewed routinely by a combination of clinical assessment and CT or fluorodeoxyglucose-PET (FDG-PET) before the administration of chemotherapy. FDG-PET was often used as an interim scan, and the metabolic tumor volume (MTV) was determined from the initial and interim PET images using PET Edge software 
(MIMSoftware Inc., Cleveland, OH, USA). Serial ctDNA profiling was conducted during the patient's therapy course.

\section{Sample collection and DNA extraction}

Before treatment, peripheral blood samples were collected using $10 \mathrm{ml}$ EDTA vacutainer tubes and processed within $4 \mathrm{~h}$ at the constant temperature of $4{ }^{\circ} \mathrm{C}$. cfDNA was extracted from plasma using the QIAamp Circulating Nucleic Acid Kit (Qiagen, Valencia, California) following the manufacturer's instructions. Genomic DNA was extracted from peripheral mononuclear cells and formalin fixed paraffin-embedded (FFPE) tissue. The DNA concentration and quality were estimated using a Qubit fluorometer (Invitrogen). The cfDNA quality was assessed using an Agilent 2100 Bioanalyzer and DNA HS Kit (Agilent Technologies, Palo Alto, CA, USA).

\section{Multiregional targeted NGS of patients' plasma and tumor sample}

Sequencing was performed on FFPE tumor DNA, plasma cfDNA and PBMC, and targeted sequencing gene panels including the coding exons and splice sites of 41 genes (Yuanqi Biopharmaceutical Co., Shanghai, China) that are recurrently mutated in NK/T-cell lymphoma were specifically designed for this study, with the genes including $A D A M 3 A, A P C, A R I D 1 A, A R I D 1 B$, ARID2, ASXL3, ATM, BCOR, BCORL1, CD28, CHD8, CREBBP, DDX3X, DNMT3A, EP3O0, EZH2, FYN, IDH2, IL2RG, JAK1, JAK3, KDM6A, KMT2A, KMT2D, MGA, NF1, NOTCH1, PRDM1, PTPN1, RHOA, SETD2, SOCS1, STAT3, STAT5B, STAT6, TET1, TET2, TNFRSF14, TP53, TRAF3 and ZAP608. Tumor DNA was sheared through sonication before library construction to obtain an almost 200-bp fragment of cfDNA, which possesses a fragmented DNA nature. No additional fragmentation was performed before library construction. NGS libraries were constructed using the SureSelect Library Prep Kit (Agilent Technologies, Palo Alto, CA, USA). Quantification of the library was performed using the Agilent DNA 1000 Kit (Agilent Technologies). Sequencing was performed on the Illumina MiSeq system (Illumina, San Diego, CA) following the manufacturer's protocol. For tumor DNA, the mean depth of each sample was $2500 \times$. Lengths of the cfDNA fragments primarily ranged from 100 to $200 \mathrm{bp}$, and the average coverage depths for sequencing was $813.42 \times$ (range, $462 \times-6513 \times$ ), with an average of $5 \%$ of the target sequence being covered at sufficient depth for variant calling. Bioinformatics analysis was performed to verify the sample sequence and mutation site and to calculate the mutated allele frequency (MAF) compared with the human genome sequence (hg19) using Burrows-Wheel Aligner (BWA) sequence alignment software. Samtools version 1.3 was used for single nucleotide variant (SNV)/indel calling and filter workflow.

\section{Statistical analysis}

Statistical analysis was conducted using SPSS software (Version 18.0, LEAD Corp). Descriptive statistics were used to analyze the clinical, demographic and genetic test result characteristics. Concentrations of ctDNA were expressed in haploid genome equivalents per $\mathrm{mL}$ (hGE/ $\mathrm{mL}$ ) and were calculated by multiplying the mean ctDNA mutant allele frequencies by the input concentration of cfDNA in $\mathrm{pg} / \mathrm{mL}$ as determined by fluorometry and then dividing by 3.3 [20]. The correlation of gene mutation, ctDNA concentration and clinicopathologic features of patients were conducted using the Pearson $X^{2}$ tests. The overall survival (OS) measured the proportion of patients who were alive at a specific time after diagnosis. Survival estimates were obtained using the Kaplan-Meier method, and comparisons were made using a log-rank test. Unpaired Student's t-test for two groups was used in this study. A COX proportional regression model was used to calculate the survival hazard ratio (HR). Statistical differences were considered significant if the $P$ value was less than 0.05 .

\section{Results}

\section{Patient characteristics}

To examine the feasibility of ctDNA detection in plasma, from February 2017 to December 2019, 65 newly diagnosed ENKTL patients were recruited, and initial and longitudinal plasma specimens were obtained from patients undergoing chemotherapy (Fig. 1). The median age at first blood draw was 45.2 years (range, 40 to 55 years), and 45 patients were men. At disease onset, 26 patients (40\%) exhibited B symptoms. According to the Ann Arbor staging criteria, 14 patients were identified as stage I, 20 patients were identified as stage II, 13 patients were identified as stage III, and 18 patients were identified as stage IV (Table 1). ctDNA was successfully extracted from all patients and healthy individuals. The average concentration of the ctDNA was $3.79 \mathrm{hEG} / \mathrm{ml}$ (range, 0 to $5.39 \mathrm{hEG} / \mathrm{ml})$.

\section{ctDNA mutation spectrum of newly diagnosed ENKTL}

Of the 65 patients recruited in this study, to exclude hematopoietic clonal mutations, somatic mutations observed in both plasma and PBMC were excluded, mutation were detected in the plasma of 49 (75.3\%) patients with a median of 1.7 per sample (range, 0 to 19) (Fig. 2, upper panels). The range of mutant allele frequencies in each gene of the samples was shown in Table S1. The most frequently mutated genes were KMT2D (23.1\%), 


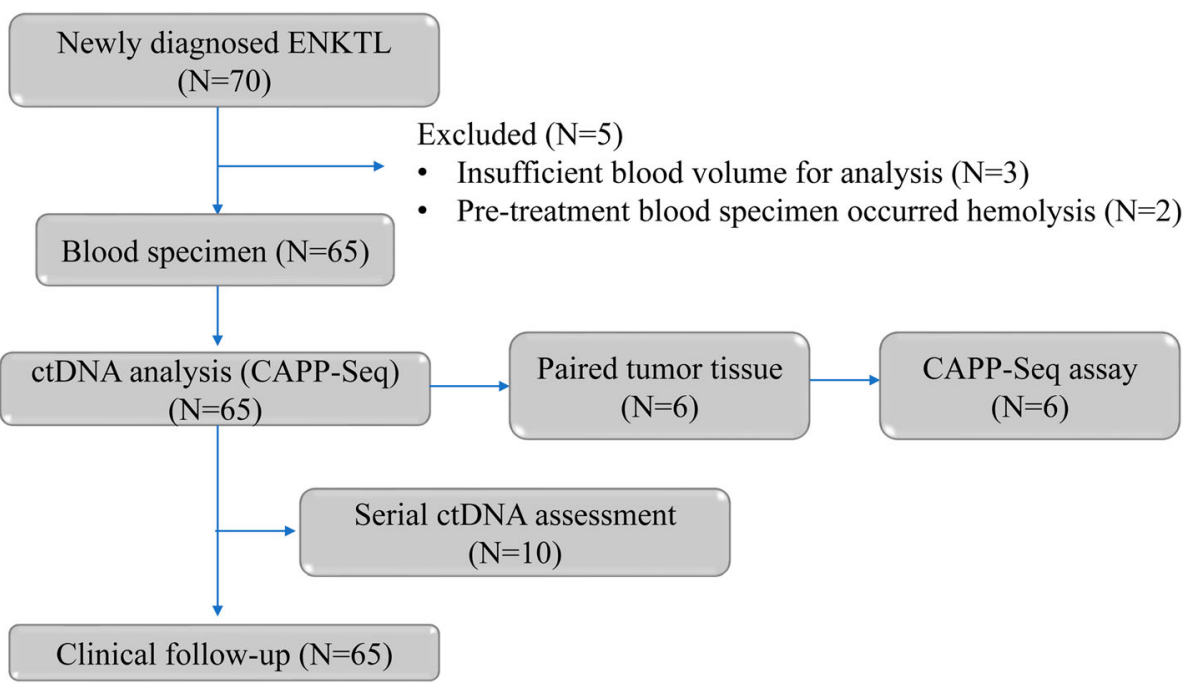

Fig. 1 Consort diagram of patient enrolment and specimen collection

APC (12.3\%), ATM (10.8\%), ASXL3 (9.2\%), JAK3 (9.2\%), SETD2 (9.2\%), TP53 (9.2\%) and NOTCH1 (7.7\%) (Fig. 2, middle panels). Consistent with the somatic SNV spectrum in other tumors, we found that $C>T / G>A$ was a preferred alteration (Fig. 2, lower panels).

Table 1 Patients' demographic and clinical characteristics

\begin{tabular}{|c|c|c|}
\hline Prognostic variables & No. & Proportion(\%) \\
\hline \multicolumn{3}{|l|}{ Gender } \\
\hline Male & 45 & 69.23 \\
\hline Female & 20 & 30.77 \\
\hline \multicolumn{3}{|l|}{ Clinical stage } \\
\hline । & 14 & 21.54 \\
\hline$\|$ & 20 & 30.77 \\
\hline$\|$ & 13 & 20.00 \\
\hline IV & 18 & 27.69 \\
\hline \multicolumn{3}{|l|}{ B symptoms } \\
\hline With & 26 & 40.00 \\
\hline Without & 39 & 60.00 \\
\hline \multicolumn{3}{|l|}{ IPI Scores } \\
\hline 1 & 22 & 33.85 \\
\hline 2 & 24 & 36.92 \\
\hline 3 & 14 & 21.54 \\
\hline 4 & 5 & 7.69 \\
\hline \multicolumn{3}{|c|}{ Final Recurrence status } \\
\hline With & 9 & 13.85 \\
\hline Without & 56 & 86.15 \\
\hline \multicolumn{3}{|l|}{ Ki67 index } \\
\hline Low & 33 & 50.77 \\
\hline High & 32 & 49.23 \\
\hline
\end{tabular}

\section{Correlation of detectable ctDNA with the clinical} characteristics of ENKTL patients

To assess the concordance of the ctDNA test with the tissue NGS test, 6 paired tumor biopsies were genotyped. Compared with the results of the plasma ctDNA spectrum, most of the genes overlapped, the average biopsy-confirmed detection ratio was 93.75\% (range 75100\%) (Fig. 3a). In 4 patients, biopsy-confirmed tumor mutations were all detectable in ctDNA samples (Fig. 3b), but for patient \#10, TRAF3 variation was not detected in tumor DNA. For patient \#8, STAT3 could not be detected in tumor ctDNA (Fig. 3c). These differences might be caused by tumor anatomical heterogeneity or tumorassociated stromal tissue infiltration.

To validate the potential clinical utility of the mutations detected in the plasma ctDNA, we investigated the correlation between clinical factors and ctDNA levels. As MTV measured by PET/CT could quantitatively reflect the tumor burden, we found that the plasma ctDNA concentration was significantly correlated with the MTV $(P=0.04)$ (Fig. 4a), suggesting that ctDNA could be identified as a promising biomarker of the tumor load. In patients with stage III-IV disease, the mutation frequencies of KMT2D (11/33), ATM (5/33), and $J A K 3(3 / 33)$ were higher than that of patients with stage I-II disease. Additionally, mutation allele frequencies of KMT2D, $A P C$ and $A S X L 3$ were not correlated with disease stage, but the mutation allele frequencies of $A T M$ and JAK3 showed significant differences (Fig. 4b). Furthermore, the correlation of the mutation status of each gene with the metabolic tumor burden was investigated. We found that patients with mutated KMT2D, ASXL3, and JAK3 showed significantly higher MTV than patients with 


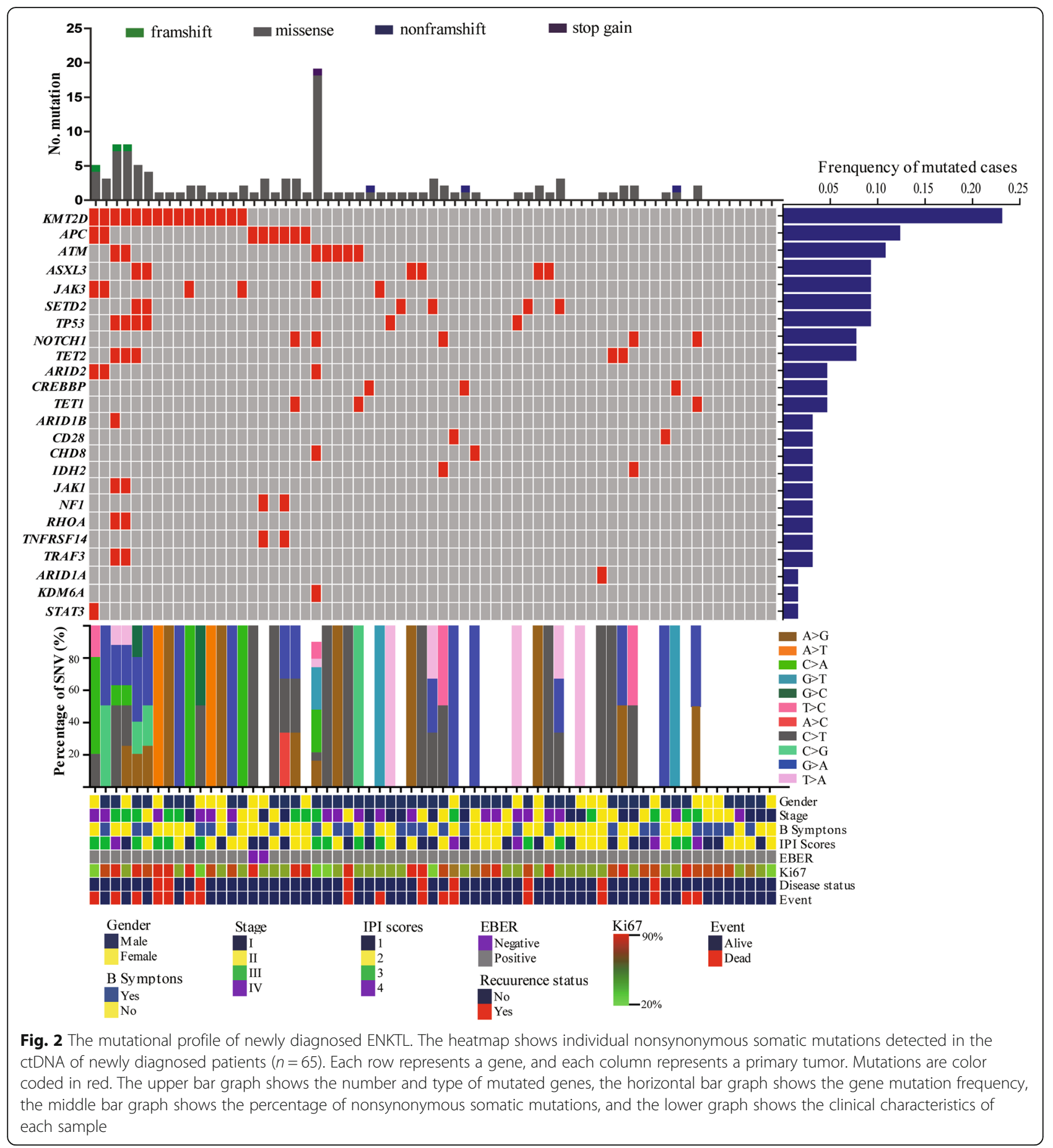

wild type (Fig. 4d), suggesting that these genes might be positively correlated with the disease malignancy. Recently, plasma EBV-DNA has served as a valuable biomarker of the tumor load and prognostic factor, thus, we also evaluated the correlation between the plasma EBV-DNA and clinical characteristics, and found that the pre-treatment plasma EBV-DNA was positively correlated with MTV (Fig. 4c), but the correlation between EBV-DNA levels and the ctDNA concentration were not significant different (Fig. 4e).

Serial ctDNA detection during therapy could complement the response assessment of the patients, and patients with mutated KMT2D and ATM predicted poor prognosis We further investigated the potential role of plasma ctDNA in the therapeutic monitoring of patients 


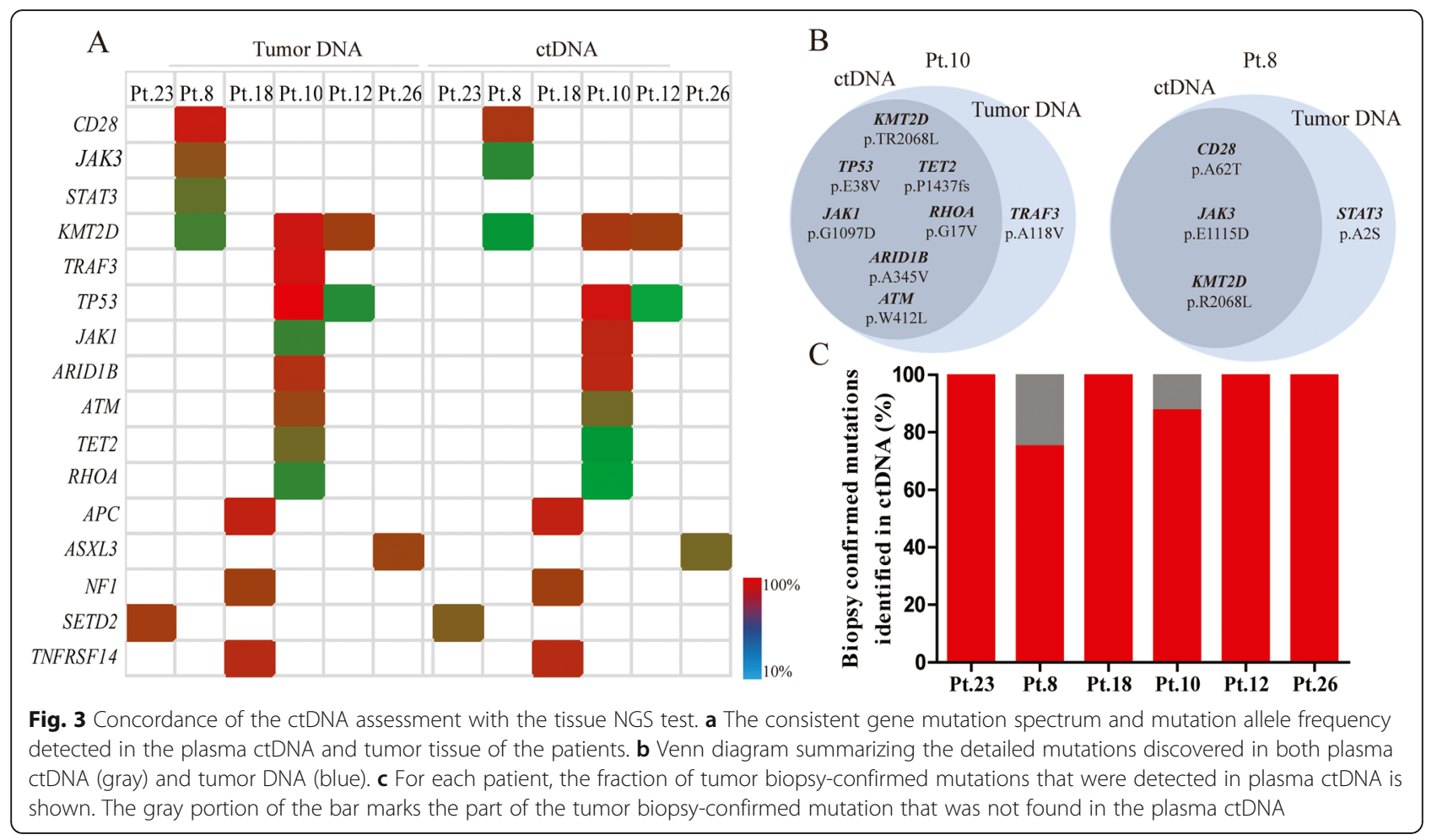

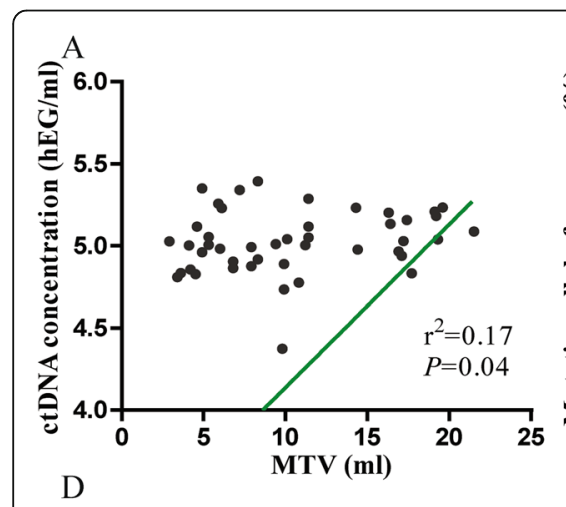

D

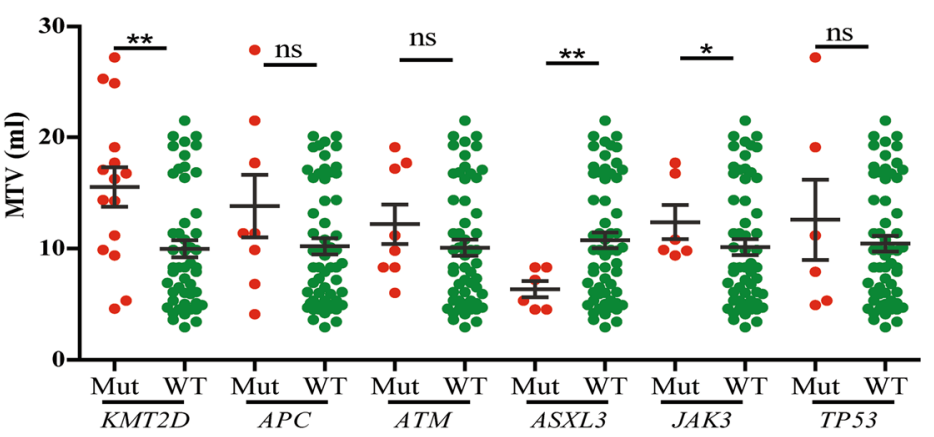

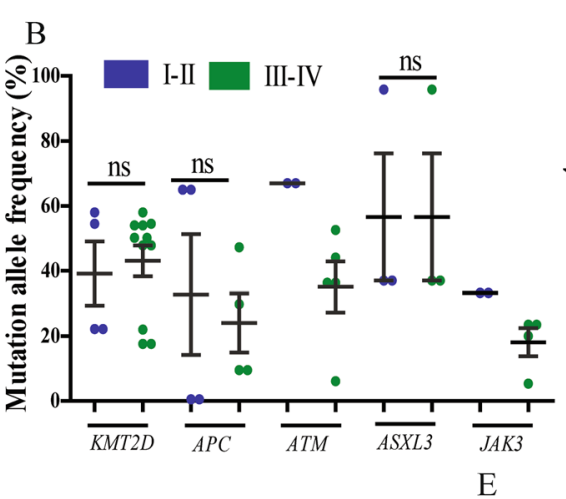

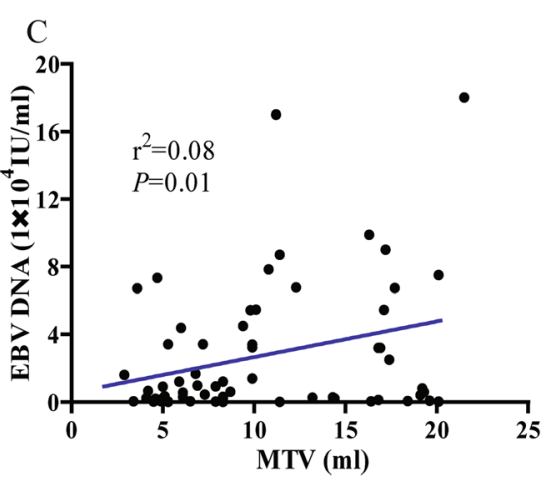

$\mathrm{E}$

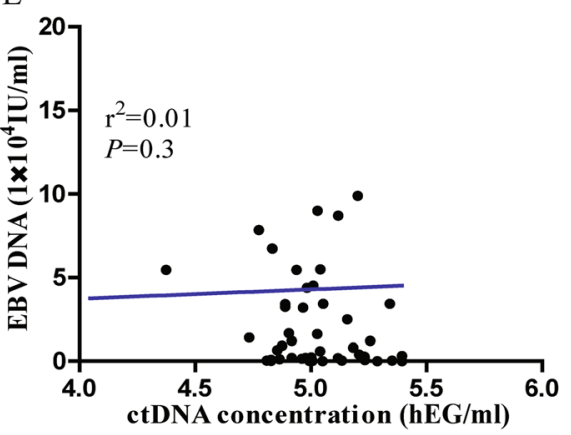

Fig. 4 Correlation of the ctDNA assessment with the patient clinical characteristics. a Linear regression of the plasma ctDNA concentration with metabolic tumor volume. b Mutation allele frequencies of KMT2D, APC, ATM, ASXL3 and JAK3 in patients with different tumor stages. c The correlation between plasma EBV-DNA levels and metabolic tumor volume. $\mathbf{d}$ The general metabolic tumor volume in patients with different mutation statuses of KMT2D, APC, ATM, ASXL3, JAK3 and TP53. e The correlation of the EBV-DNA level with the ctDNA concentration 
undergoing chemotherapy. Ten patients were recruited, and sequential plasma samples upon disease diagnosis, before treatment cycle 4 (C4), and before cycle 8 (C8) were collected. In total, the mutated gene number and mutation allele frequencies decreased with the chemotherapy administration, 7 patients could achieve CR at C4, in these patients, only 2 patients didn't detect ctDNA mutations at $\mathrm{C} 4$, suggesting that ctDNA assessment was more sensitive than PET/CT scans, all patients couldn't detect plasma EBV-DNA when achieved CR. In addition, with more cycles of chemotherapy, all patients could achieve complete molecular remission (Fig. 5). In these 10 patients, we also observed one relapsed, and the relapsed mutation spectrum was similar to the original spectrum. All these results suggested that ctDNA detection could be regarded as a molecular response evaluation marker, and could complement the response assessment of the patients.

As $K M T 2 D$ was the most frequently somatic mutations, the expression of KMT2D was investigated, and we found that the KMT2D expressions of patients with mutations were higher than that of patients with wild type (Fig. 6a). Then, prognosis value of the plasma ctDNA mutation status and EBV-DNA level was also investigated. KaplanMeier analysis estimated that patients with mutated KMT2D and ATM had a shorter OS (Fig. 6b, c), but no significant difference was observed in patients with other mutated genes (Fig. 6d-g), which might be due to the small sample recruited in our cohort. In addition, patients with high EBV-DNA levels showed significantly poorer prognosis (Fig. 6h). The results of univariate and multivariate analysis for risk factor of OS in patients were summarized in Table 2. Univariate analysis of the factors revealed that the stage, IPI scores, recurrence status, EBVDNA levels, KMT2D mutation and ATM mutation were independent prognostic indicators of the overall survival of patients, and multivariate analysis showed that the EBV-DNA level, KMT2D mutation and ATM mutation were solely prognostic factors. All these results suggested that the dynamic detection of ctDNA during therapy could better reflect the disease status than PET/CT scan, and patients with mutated $K M T 2 D$, and $A T M$ predicted poor prognosis.

\section{Discussion}

This study was the first to determine the plasma ctDNA gene mutation spectrum of ENKTL and demonstrated that ctDNA sequencing could be considered a promising biomarker for monitoring the minimal residual disease (MRD) of patients. The most frequently mutated genes in plasma ctDNA were KMT2D (23.1\%), APC (12.3\%), ATM (10.8\%), ASXL3 (9.2\%), JAK3 (9.2\%), SETD2 (9.2\%), TP53 (9.2\%), and NOTCH1 (7.7\%). The ctDNA concentration was positively correlated with tumor stage and

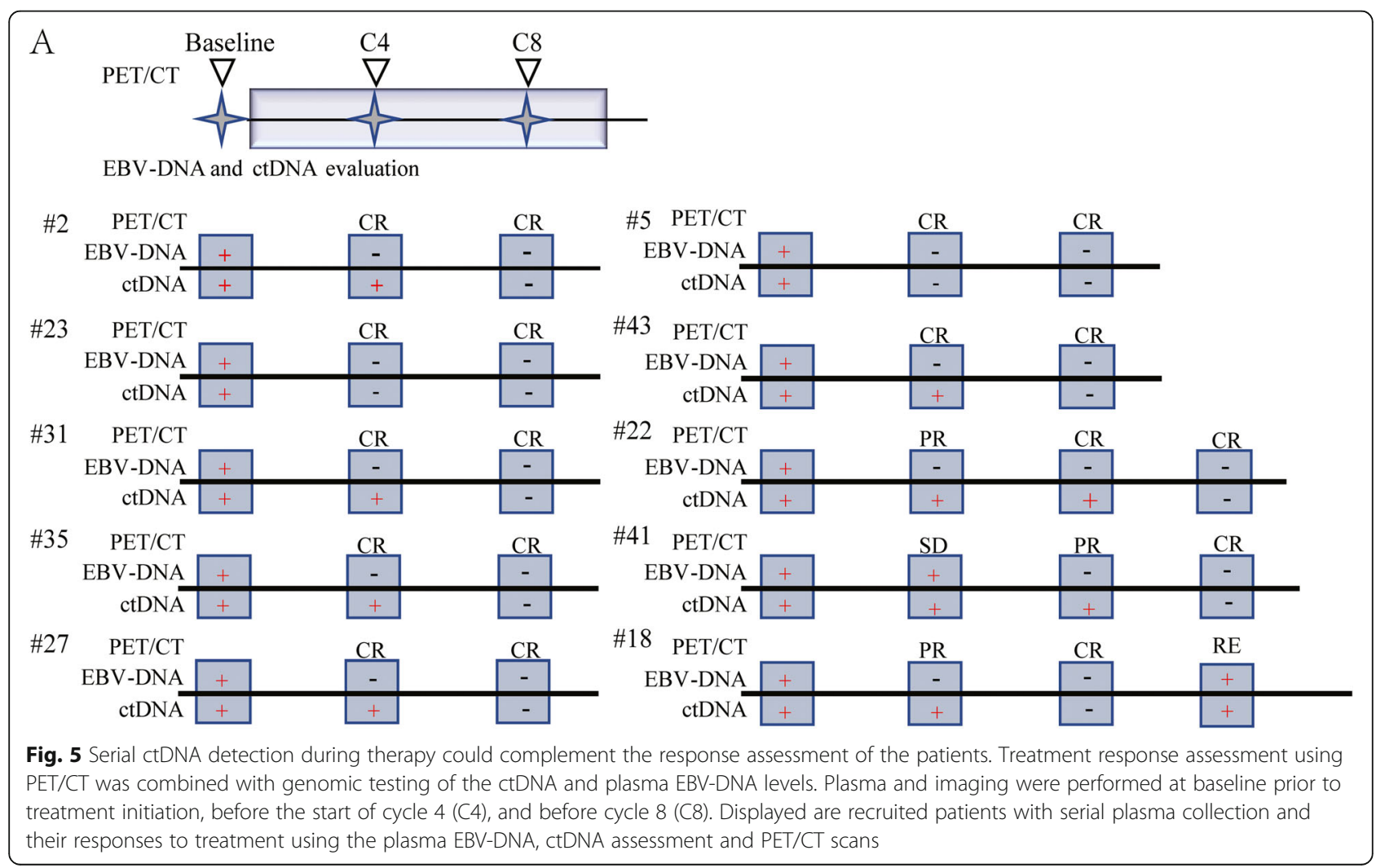




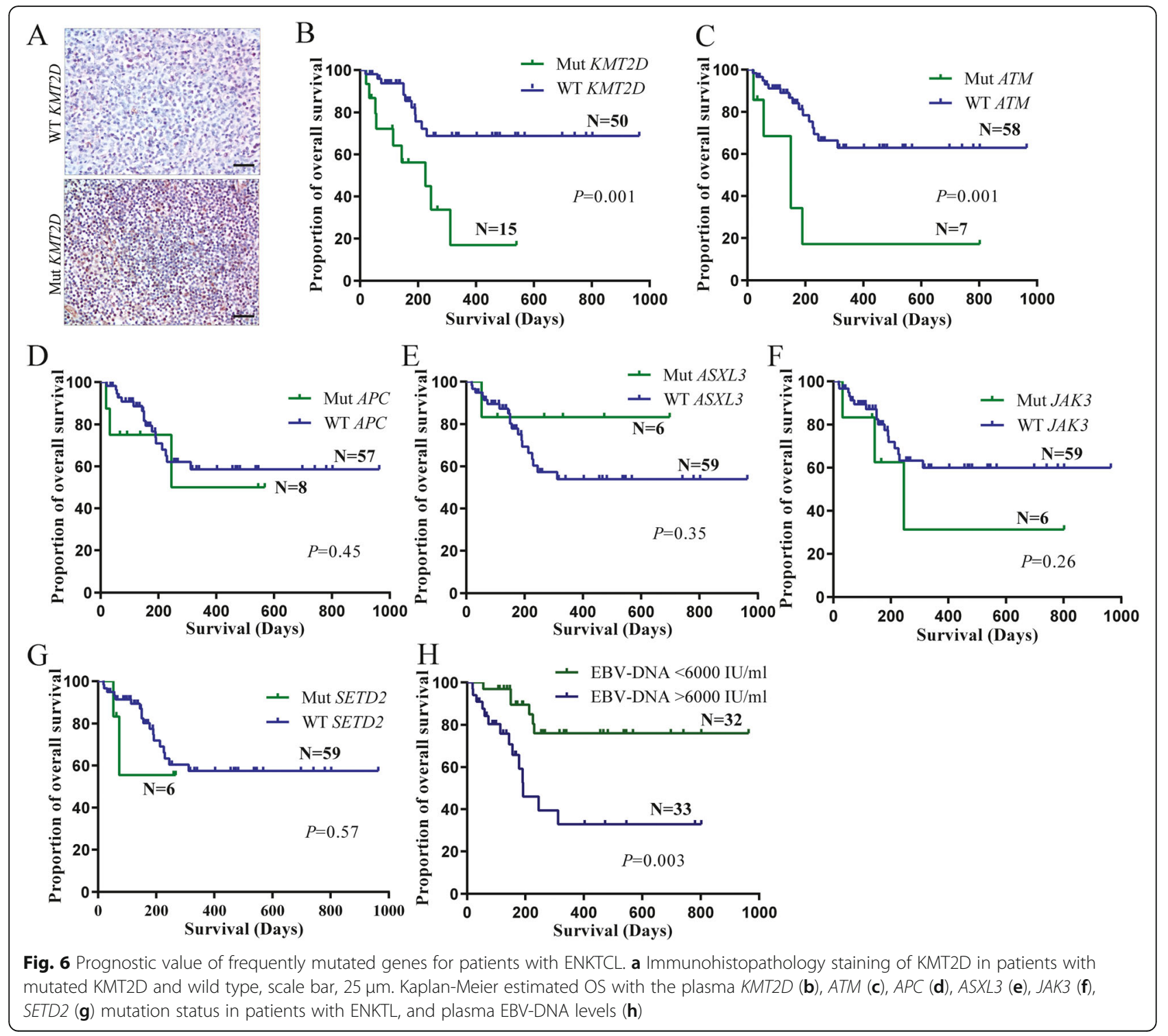

MTV. In terms of consistency with tumor tissue, almost all of the gene mutations detected in the plasma ctDNA could be found in tumor DNA, and further analysis demonstrated that a decreased number and MAF of certain genes in plasma ctDNA could complement the therapeutic response of the patients, and patients with mutated KMT2D and ATM had poor prognosis, suggesting that the gene mutations in plasma could serve as promising biomarkers for use in the diagnosis or monitoring of ENKTL disease courses.

Tumor biopsies can only offer information about lymphoma at one specific time in a specific area and can be accompanied with complications, and PET/CT may lead to false negatives due to technology limitations or false positive caused by tumor flares or pseudoprogression [27]. ctDNA detection is an optimal noninvasive and specific technique by which to overcome these traditional monitoring method limitations. A rapidly growing body of evidence has established that ctDNA detection during curative therapies can identify patients for whom there remains evidence of residual, radiographically occult cancer. In DLBCL, ctDNA can be used to predict the tumor load and treatment outcome of newly diagnosed patients, and patients with a pretreatment ctDNA level above the median had a significantly poorer prognosis than those with levels below the median [28]. Later, Kurtz et al. reported that serial ctDNA measurements of 125 patients were an effective tool for prognostication, patients with 2-log or greater decreases in ctDNA had a better event-free survival (EFS) after one cycle of therapy, and patients with 2.5-log or greater reduction in ctDNA had a significantly better EFS after two cycle treatments. These studies suggested that serial ctDNA detection could be used to assess the therapy 
Table 2 Univariate and multivariate analyses of the overall survival of patients

\begin{tabular}{|c|c|c|c|c|}
\hline \multirow[t]{2}{*}{ Prognostic variables } & \multicolumn{2}{|l|}{ Univariate analysis } & \multicolumn{2}{|l|}{ Multivariate analysis } \\
\hline & $\mathrm{HR}(95 \% \mathrm{Cl})$ & $p$ value & $\mathrm{HR}(95 \% \mathrm{Cl})$ & $p$ value \\
\hline Gender (female vs. male) & $0.712(0.279-1.812)$ & 0.476 & & \\
\hline Stage (I-II vs. III-IV) & $2.625(1.043-6.604)$ & 0.04 & $1.159(0.253-5.311)$ & 0.849 \\
\hline B symphony (with vs. without) & $1.234(0.512-2.973)$ & 0.64 & & \\
\hline$|\mathrm{P}|$ & $2.595(1.073-6.277)$ & 0.034 & $0.565(0.118-2.712)$ & 0.476 \\
\hline Final recurrence status & $3.346(1.364-8.204)$ & 0.008 & 2.705 (0.941-7.776) & 0.065 \\
\hline MTV & $2.069(0.843-5.078)$ & 0.112 & & \\
\hline ctDNA concentration & $2.975(0.984-8.997)$ & 0.053 & & \\
\hline EBV-DNA level & $3.672(1.396-9.664)$ & 0.008 & $3.174(1.091-9.231)$ & 0.034 \\
\hline KMT2D mutation (without vs. with) & $0.289(0.119-0.702)$ & 0.006 & $0.266(0.086-0.828)$ & 0.022 \\
\hline APC mutation (without vs. with) & $0.550(0.16-1.895)$ & 0.344 & & \\
\hline ATM mutation (without vs. with) & $0.271(0.098-0.752)$ & 0.012 & $0.212(0.064-0.700)$ & 0.011 \\
\hline ASXL3 mutation (without vs. with) & $2613(0.347-19.68)$ & 0.351 & & \\
\hline JAK3 mutation (without vs. with) & $0.569(0.166-1.949)$ & 0.369 & & \\
\hline SETD2 mutation (without vs. with) & $0.804(0.184-3.507)$ & 0.771 & & \\
\hline TP53 mutation (without vs. with) & $0.615(0.179-2.115)$ & 0.441 & & \\
\hline NOTCH1 mutation (without vs. with) & $0.410(0.120-1.406)$ & 0.156 & & \\
\hline
\end{tabular}

response dynamically and guide personalized target therapy. In another study, Daigle et al. found that mutation of $C R E B B P$ in $\mathrm{R} / \mathrm{R}$ DLBCL had the greatest association with tazemetostat treatment (an EZH2 inhibitor), and patients with mutations in PIM1, BCL6, HIST1H1E and TP53 lacked response to treatment [29]. In HL, Valeria et al. determined the molecular mutation spectrum using ctDNA measurement. The most frequently mutated genes were STAT, TNFAIP3 and ITPKB, and detection sensitivity was $87.5 \%$, suggesting that ctDNA could well reflect the spectrum of tumor tissue mutation. Compared with newly diagnosed and refractory HL mutation profiles, most mutations overlapped, and mutations of STAT, TNFAIP3, GNA13 and ITPKB might be an ancestral clone persisted throughout the disease course [23]. Although only a few studies have focused on assessing ctDNA in T-cell lymphoma, preliminary evidence has also demonstrated that ctDNA measurement in TCL is feasible [26, 30]. Our study is the first to determine the mutation spectrum of ctDNA in ENKTL. The most commonly mutated genes are KMT2D, APC, ATM, ASXL3, JAK3, SETD2, TP53 and NOTCH1. Our results revealed that ctDNA could reflect the MTV, and by longitudinally profiling patients treated with chemotherapy. In addition, we provided evidence that serial ctDNA assessment could monitor the disease status, with the results able to partially reflect which mutated gene was sensitive to chemotherapy drugs. However, only one of the 10 recruited patients relapsed. For relapsed patients, whether primary mutation clone recurrence or new mutation occupies the main role needs to be further investigated. In addition, future trials should include more patients and long-term follow-up to explore the plasma gene clone evolution process.

Plasma cfDNA includes tumor cell-derived plasma ctDNA and normal cell apoptosis-derived cfDNA, which are DNA fragments. ctDNA could be a more comprehensive reflection of gene mutations in all tumors than conventional biopsies [31, 32]. Therefore, ctDNA-based detection methods are critical for identifying which mutation are tumor-specific. As sensitivities increase with the development of new methods and because agerelated somatic mutations could also lead to hematopoietic clonal expansion, the possibility of falsepositive results might occur [33, 34]. Recently, Razavi et al. showed that clonal hematopoiesis was the major contributor to plasma variants using a high-intensity sequencing assay of matched plasma DNA, tumor tissue and white blood cells, emphasizing that matched cfDNA-white blood cells were critical for accurately screening somatic mutations [35]. In the present study, to decrease the bias caused by clonal hematopoiesis associated mutations, the age range of the included patients was narrow, matched ctDNA-PBMC sample was applied, and a sequencing panel was conducted according to a previous gene-expression profiling study on ENKTL. Zhao et al. confirmed that the most frequently mutated genes in NKTCL were the RNA helicase gene $D D X 3 X$, tumor suppressors (TP53 and MGA), JAKSTAT pathway molecules (STAT3 and STAT5b) and epigenetic modifiers (MLL2, ARID1A, EP300 and $A S X L 3)$ [36, 37]. In addition to these recurrent 
mutations, JAK3, KMT2D, EZH2, NOTCH1 and TET2 were also identified by different groups [38-41]. Based on these findings combined with the most frequent gene mutation spectrum in Chinese individuals, we selected 41 mutated genes for our study. Interestingly, we found that KMT2D alterations possessed a higher frequency (23.1\%) and which were all non-synonymous mutations (p.R2068L, p.D4378E, p.R755W, p.P2382S, p.V160L, p.L2316V). We also found some mutation existed in both plasma and PBMC, such as MGA (p.T716S, p.P1523A), ASXL3 (p.N954S), EZH2 (p.D185H), TET2 (p. P29R), $P 53$ (p.P72R), APC (p.V1822D), KDM6A (p.T726K), et al. KMT2D belongs to a family of mammalian histone $\mathrm{H} 3$ lysine 4 (H3K4) methyltransferase. It is frequently mutated in Kabuki syndrome and various cancers, and it acts as a scaffold protein within the complex depending on the cell type and stage of differentiation [42]. In acute myeloid leukemia, follicular lymphoma and DLBCL, KMT2D could inhibit tumorigenesis and metastasis [43, 44], but in solid cancer, such as prostate cancer, KMT2D is critical factor for promoting tumor cell proliferation [45]. In our study, we found that the KMT2D expression of patients with the mutation was higher than that of patients with wild type, suggesting that KMT2D might be an oncogene in NKTCL lymphoma. However, its underlying significance in NKTCL has not been explored to date, and further studies regarding the function of KMT2D mutation in NKTCL lymphomagenesis are urgently recommended.

\section{Conclusions}

To our knowledge, this is the first study to prospectively evaluate the potential utility of ctDNA analysis for NKTCL patients. We have demonstrated that ctDNA assessment could predict the therapy response in NKTCL, and the highest mutation frequencies were in $K M T 2 D$, APC, ATM, ASXL3, JAK3, SETD2, TP53, NOTCH1. Our results could define the optimal strategy for patient follow-up.

\section{Supplementary information}

Supplementary information accompanies this paper at https://doi.org/10. 1186/s40364-020-00205-4.

Additional file 1: Table S1. The mutant allele frequencies in each gene of each sample.

Additional file 2: Table S2. Correlation between KMT2D mutation status and clinicopathological features of ENKTL.

Additional file 3: Table S3. Correlation between ATM mutation status and clinicopathological features of ENKTL.

\section{Abbreviations}

ENTKL: Extranodal NK/T-cell lymphoma, nasal type; CT: Computed tomography; Non-NHL: Non-Hodgkin lymphoma; cfDNA: Circulating cell-free DNA; ctDNA: Circulating tumor DNA; DLBCL: Diffuse large B-cell lymphoma; MTV: Metabolic tumor volume; ADAM3A: ADAM Metallopeptidase Domain
3A; APC: Adenomatous Polyposis Coli Protein; ARID1A: AT-Rich Interaction Domain 1A; ARID1B: AT-Rich Interaction Domain 1B; ARID2: AT-Rich Interaction Domain 2; ASXL3: ASXL Transcriptional Regulator 3; ATM: Ataxia Telangiectasia Mutated; BCOR: BCL6 Corepressor; BCORL 1: BCL6 Corepressor Like 1; CHD8: Chromodomain Helicase DNA Binding Protein 8; CREBBP: CREB Binding Protein; DDX3X: DEAD-Box Helicase 3 X-Linked; DNMT3A: DNA Methyltransferase 3 Alpha; EP300: E1A Binding Protein P300; EZH2: Enhancer of Zeste 2 Polycomb Repressive Complex 2 Subunit; FYN: Src Family Tyrosine Kinase; IDH2: Isocitrate Dehydrogenase (NADP (+)) 2, Mitochondrial; IL2RG: Interleukin 2 Receptor Subunit Gamma; JAK1: Janus Kinase 1; JAK3: Janus Kinase3; KDM6A: Lysine Demethylase 6A; KMT2A: Lysine Methyltransferase 2A; KMT2D: Lysine Methyltransferase 2D; MGA: MAX Dimerization Protein; NF1: Neurofibromin 1; NOTCH1: Notch Receptor 1; PRDM1: Positive Regulatory Domain I-Binding Factor 1; PTPN1: Protein Tyrosine Phosphatase Non-Receptor Type 1; RHOA: Ras Homolog Family Member A; SETD2: SET Domain Containing 2; SOCS1: Suppressor of Cytokine Signaling 1; STAT3: Signal Transducer and Activator of Transcription 3; STAT5B: Signal Transducer and Activator of Transcription 5B; STAT6: Signal Transducer and Activator of Transcription 6; TET1: Tet Methylcytosine Dioxygenase 1; TNFRSF14: TNF Receptor Superfamily Member 14; TP53: Tumor Protein P53; TRAF3: TNF Receptor Associated Factor 3; ZAP608: Zeta Chain Of T Cell Receptor Associated Protein Kinase 608; MAF: Mutated allele frequency; SNV: Single nucleotide variant

\section{Acknowledgments}

We thank Dr. Yimei Feng for their assistance with the statistical analysis and Qing Xu for her encouragement. The authors also thank their colleagues for helpful comments.

\section{Authors' contributions}

Jun Rao, Yao Liu and Xi Zhang conceived and designed the study. Jun Rao, Wei Zhang and Qiong Li developed the methodology and analyzed and interpreted the data. Jun Rao and Qiong Li wrote the manuscript. All authors reviewed and revised the manuscript. The author(s) read and approved the final manuscript.

\section{Funding}

This project was supported by grants from the National Natural Science Fund for Youth (No.81600166), the technique innovation and applied program of Chongqing (cstc2018jscx-msybX0052), the Scientific and Technological Innovation Program of Chongqing social undertakings and people's livelihood guarantee (cstc2016shms-ztzx10003,

cstc2017shmsA130003) and Science and technology innovation improvement project of AMU (2019XLC3020).

Availability of data and materials

All data generated and analyzed during this study are included in this article and its supplementary information files.

Ethics approval and consent to participate

The study was approved by China Ehics Committees of Registering Clinical Trials (ChiECRCT-2018005).

Consent for publication

Not applicable.

\section{Competing interests}

The authors declare that they have no competing interests.

\section{Author details}

${ }^{1}$ Medical Center of Hematology, Xinqiao Hospital, Army Medical University, Chongqing, China. 'State Key Laboratory of Trauma, Burns and Combined Injury, Army Medical University, Chongqing 400037, China.

Received: 22 May 2020 Accepted: 6 July 2020

Published online: 17 July 2020

References

1. Tse E, Kwong YL. How I treat NK/T-cell lymphomas. Blood. 2013;121(25): 4997-5005. 
2. Xiong J, Zhao W. What we should know about natural killer/T-cell lymphomas. Hematol Oncol. 2019;37(Suppl 1):75-81.

3. Kim SJ, Yoon SE, Kim WS. Treatment of localized extranodal NKTT cell lymphoma, nasal type: a systematic review. J Hematol Oncol. 2018;11(1):140.

4. Liu W, Liu J, Song Y, Wang X, Zhou M, Wang L, Ma J, Zhu J. Mortality of lymphoma and myeloma in China, 2004-2017: an observational study. J Hematol Oncol. 2019;12(1):22.

5. Kwong YL, Kim WS, Lim ST, Kim SJ, Tang T, Tse E, Leung AY, Chim CS. SMILE for natural killer/T-cell lymphoma: analysis of safety and efficacy from the Asia Lymphoma Study Group. Blood. 2012;120(15):2973-80.

6. Au WY, Weisenburger DD, Intragumtornchai T, Nakamura S, Kim WS, Sng I, Vose J, Armitage JO, Liang R. Clinical differences between nasal and extranasal natural killer/T-cell lymphoma: a study of 136 cases from the international peripheral T-cell lymphoma project. Blood. 2009;113(17):3931-7.

7. Somasundaram N, Lim JQ, Ong CK, Lim ST. Pathogenesis and biomarkers of natural killer T cell lymphoma (NKTL). J Hematol Oncol. 2019;12(1):28.

8. Adams HJ, Nievelstein RA, Kwee TC. Prognostic value of complete remission status at end-of-treatment FDG-PET in R-CHOP-treated diffuse large B-cell lymphoma: systematic review and meta-analysis. Br J Haematol. 2015;170(2): 185-91.

9. Trotman J, Luminari S, Boussetta S, Versari A, Dupuis J, Tychyj C, Marcheselli L, Berriolo-Riedinger A, Franceschetto A, Julian A, et al. Prognostic value of PET-CT after first-line therapy in patients with follicular lymphoma: a pooled analysis of central scan review in three multicentre studies. Lancet Haematol. 2014;1 (1):e17-27.

10. Kim SJ, Choi JY, Hyun SH, Ki CS, Oh D, Ahn YC, Ko YH, Choi S, Jung SH, Khong PL, et al. Risk stratification on the basis of Deauville score on PET-CT and the presence of Epstein-Barr virus DNA after completion of primary treatment for extranodal natural killer/T-cell lymphoma, nasal type: a multicentre, retrospective analysis. Lancet Haematol. 2015;2(2):e66-74.

11. Forshew T, Murtaza M, Parkinson C, Gale D, Tsui DW, Kaper F, Dawson SJ, Piskorz AM, Jimenez-Linan M, Bentley D, et al. Noninvasive identification and monitoring of cancer mutations by targeted deep sequencing of plasma DNA. Sci Transl Med. 2012;4(136):136ra168.

12. Bettegowda C, Sausen M, Leary RJ, Kinde I, Wang Y, Agrawal N, Bartlett BR, Wang H, Luber B, Alani RM, et al. Detection of circulating tumor DNA in early- and late-stage human malignancies. Sci Transl Med. 2014;6(224): $224 \mathrm{ra} 224$.

13. Siravegna G, Marsoni S, Siena S, Bardelli A. Integrating liquid biopsies into the management of cancer. Nat Rev Clin Oncol. 2017;14(9):531-48.

14. Crowley E, Di Nicolantonio F, Loupakis F, Bardelli A. Liquid biopsy: monitoring cancer-genetics in the blood. Nat Rev Clin Oncol. 2013;10(8): 472-84.

15. Patel H, Okamura R, Fanta P, Patel C, Lanman RB, Raymond VM, Kato S, Kurzrock R. Clinical correlates of blood-derived circulating tumor DNA in pancreatic cancer. J Hematol Oncol. 2019;12(1):130.

16. Zhou J, Huang A, Yang XR. Liquid biopsy and its potential for management of hepatocellular carcinoma. J Gastrointest Cancer. 2016;47(2):157-67.

17. Esposito A, Criscitiello C, Locatelli M, Milano M, Curigliano G. Liquid biopsies for solid tumors: understanding tumor heterogeneity and real time monitoring of early resistance to targeted therapies. Pharmacol Ther. 2016; 157:120-4.

18. Chen Y, George AM, Olsson E, Saal LH. Identification and use of personalized genomic markers for monitoring circulating tumor DNA. Methods Mol Biol. 2018;1768:303-22.

19. Roschewski M, Dunleavy K, Pittaluga S, Moorhead M, Pepin F, Kong K, Shovlin M, Jaffe ES, Staudt LM, Lai C, et al. Circulating tumour DNA and CT monitoring in patients with untreated diffuse large B-cell lymphoma: a correlative biomarker study. Lancet Oncol. 2015;16(5):541-9.

20. Scherer F, Kurtz DM, Newman AM, Stehr H, Craig AF, Esfahani MS, Lovejoy AF, Chabon JJ, Klass DM, Liu CL, et al. Distinct biological subtypes and patterns of genome evolution in lymphoma revealed by circulating tumor DNA. Sci Transl Med. 2016;8(364):364ra155.

21. Suehara Y, Sakata-Yanagimoto M, Hattori K, Nanmoku T, Itoh T, Kaji D, Yamamoto G, Abe Y, Narita K, Takeuchi M, et al. Liquid biopsy for the identification of intravascular large B-cell lymphoma. Haematologica. 2018; 103(6):e241-4.

22. Delfau-Larue MH, van der Gucht A, Dupuis J, Jais JP, Nel I, Beldi-Ferchiou A, Hamdane S, Benmaad I, Laboure G, Verret B, et al. Total metabolic tumor volume, circulating tumor cells, cell-free DNA: distinct prognostic value in follicular lymphoma. Blood Adv. 2018;2(7):807-16.
23. Spina V, Bruscaggin A, Cuccaro A, Martini M, Di Trani M, Forestieri G, Manzoni M, Condoluci A, Arribas A, Terzi-Di-Bergamo L, et al. Circulating tumor DNA reveals genetics, clonal evolution, and residual disease in classical Hodgkin lymphoma. Blood. 2018;131(22):2413-25.

24. Liu J, Liu W, Mi L, Zeng X, Cai C, Ma J, Wang L. Incidence and mortality of multiple myeloma in China, 2006-2016: an analysis of the global burden of disease study 2016. J Hematol Oncol. 2019;12(1):136.

25. Manier S, Park J, Capelletti M, Bustoros M, Freeman SS, Ha G, Rhoades J, Liu CJ, Huynh D, Reed SC, et al. Whole-exome sequencing of cell-free DNA and circulating tumor cells in multiple myeloma. Nat Commun. 2018;9(1):1691.

26. Melani C, Pittaluga S, Yee L, Lucas A, Shovlin M, Jacob A, Chan K, Vignali M, Jaffe ES, Wilson WH, et al. Next-generation sequencing based monitoring of circulating-tumor DNA in untreated peripheral T-cell lymphoma. Blood. 2017;130(Suppl 1):2728.

27. Cheson BD, Ansell S, Schwartz L, Gordon LI, Advani R, Jacene HA, Hoos A, Barrington SF, Armand P. Refinement of the Lugano classification lymphoma response criteria in the era of immunomodulatory therapy. Blood. 2016;128(21):2489-96.

28. Kurtz DM, Green MR, Bratman SV, Scherer F, Liu CL, Kunder CA, Takahashi K Glover C, Keane C, Kihira S, et al. Noninvasive monitoring of diffuse large Bcell lymphoma by immunoglobulin high-throughput sequencing. Blood. 2015;125(24):3679-87.

29. Daigle S, McDonald AA, Morschhauser F, Salles G, Ribrag V, McKay P, Tilly H, Schmitt A, Gerecitano J, Fruchart C, et al. Discovery of candidate predictors of response to Tazemetostat in diffuse large B-cell lymphoma and follicular lymphoma using NGS technology on ctDNA samples collected pretreatment. Blood. 2017;130(Suppl 1):4013.

30. Sakata-Yanagimoto M, Nakamoto-Matsubara R, Komori D, Nguyen TB, Hattori K, Nanmoku T, Kato T, Kurita N, Yokoyama Y, Obara N, et al. Detection of the circulating tumor DNAs in angioimmunoblastic T- cell lymphoma. Ann Hematol. 2017;96(9):1471-5.

31. Herrera AF, Armand P. Minimal residual disease assessment in lymphoma: methods and applications. J Clin Oncol. 2017;35(34):3877-87.

32. Scherer F, Kurtz DM, Diehn M, Alizadeh AA. High-throughput sequencing for noninvasive disease detection in hematologic malignancies. Blood. 2017; 130(4):440-52.

33. Genovese G, Kahler AK, Handsaker RE, Lindberg J, Rose SA, Bakhoum SF, Chambert K, Mick E, Neale BM, Fromer M, et al. Clonal hematopoiesis and blood-cancer risk inferred from blood DNA sequence. N Engl J Med. 2014 371(26):2477-87.

34. Jaiswal S, Fontanillas P, Flannick J, Manning A, Grauman PV, Mar BG Lindsley RC, Mermel CH, Burtt N, Chavez A, et al. Age-related clonal hematopoiesis associated with adverse outcomes. N Engl J Med. 2014; 371(26):2488-98.

35. Razavi P, Li BT, Brown DN, Jung B, Hubbell E, Shen R, Abida W, Juluru K, De Bruijn I, Hou C, et al. High-intensity sequencing reveals the sources of plasma circulating cell-free DNA variants. Nat Med. 2019;25(12):1928-37.

36. Jiang L, Gu ZH, Yan ZX, Zhao X, Xie YY, Zhang ZG, Pan CM, Hu Y, Cai CP, Dong $Y$, et al. Exome sequencing identifies somatic mutations of DDX3X in natural killer/T-cell lymphoma. Nat Genet. 2015;47(9):1061-6.

37. Xiong J, Zhao WL. Advances in multiple omics of natural-killer/T cell lymphoma. J Hematol Oncol. 2018;11(1):134.

38. Koo GC, Tan SY, Tang T, Poon SL, Allen GE, Tan L, Chong SC, Ong WS, Tay K, Tao M, et al. Janus kinase 3-activating mutations identified in natural killer/ T-cell lymphoma. Cancer Discov. 2012;2(7):591-7.

39. Dobashi A, Tsuyama N, Asaka R, Togashi Y, Ueda K, Sakata S, Baba S, Sakamoto K, Hatake K, Takeuchi K. Frequent BCOR aberrations in extranodal NKT-cell lymphoma, nasal type. Genes Chromosomes Cancer. 2016;55(5):460-71.

40. Küçük C, Jiang B, Hu X, Zhang W, Chan JK, Xiao W, Lack N, Alkan C, Williams JC, Avery KN, et al. Activating mutations of STAT5B and STAT3 in lymphomas derived from $\gamma \delta$-T or NK cells. Nat Commun. 2015;6:6025.

41. Viré E, Brenner C, Deplus R, Blanchon L, Fraga M, Didelot C, Morey L, Van Eynde A, Bernard D, Vanderwinden JM, et al. The Polycomb group protein EZH2 directly controls DNA methylation. Nature. 2006;439(7078):871-4.

42. Calo E, Wysocka J. Modification of enhancer chromatin: what, how, and why? Mol Cell. 2013;49(5):825-37.

43. Chen C, Liu Y, Rappaport AR, Kitzing T, Schultz N, Zhao Z, Shroff AS, Dickins RA, Vakoc CR, Bradner JE, et al. MLL3 is a haploinsufficient 7q tumor suppressor in acute myeloid leukemia. Cancer Cell. 2014;25(5):652-65.

44. Ortega-Molina A, Boss IW, Canela A, Pan H, Jiang Y, Zhao C, Jiang M, Hu D, Agirre X, Niesvizky I, et al. The histone lysine methyltransferase KMT2D 
sustains a gene expression program that represses B cell lymphoma development. Nat Med. 2015;21(10):1199-208.

45. Lv S, Ji L, Chen B, Liu S, Lei C, Liu X, Qi X, Wang Y, Lai-Han Leung E, Wang $\mathrm{H}$, et al. Histone methyltransferase KMT2D sustains prostate carcinogenesis and metastasis via epigenetically activating LIFR and KLF4. Oncogene. 2018; 37(10):1354-68.

\section{Publisher's Note}

Springer Nature remains neutral with regard to jurisdictional claims in published maps and institutional affiliations.

Ready to submit your research? Choose BMC and benefit from:

- fast, convenient online submission

- thorough peer review by experienced researchers in your field

- rapid publication on acceptance

- support for research data, including large and complex data types

- gold Open Access which fosters wider collaboration and increased citations

- maximum visibility for your research: over $100 \mathrm{M}$ website views per year

At BMC, research is always in progress.

Learn more biomedcentral.com/submissions 